\title{
Topography of Cortical Microbleeds in Alzheimer's Disease with and without Cerebral Amyloid Angiopathy: A Post-Mortem 7.0-Tesla MRI Study
}

\author{
J. De Reuck*", F. Auger, N. Durieux, V. Deramecourt, C. Cordonnier, F. Pasquier, C.A. \\ Maurage, D. Leys, R. Bordet \\ Université de Lille 2, INSERM U1171, F-59000 Lille, France
}

[Received February 11, 2015; Revised April 27, 2015; Accepted April 29, 2015]

\begin{abstract}
Cortical microbleeds (CMBs) detected on T2*-weighted gradient-echo (GRE) magnetic resonance imaging (MRI) are considered as a possible hallmark of cerebral amyloid angiopathy (CAA). The present post-mortem 7.0-tesla MRI study investigates whether topographic differences exist in Alzheimer's brains without (AD) and with CAA (AD-CAA). The distribution of CMBs in thirty-two post-mortem brains, consisting of $12 \mathrm{AD}, 8 \mathrm{AD}$-CAA and 12 controls, was mutually compared on T2*-GRE MRI of six coronal sections of a cerebral hemisphere. The mean numbers of CMBs were determined in twenty-two different gyri. As a whole there was a trend of more CMBs on GRE MRI in the prefrontal section of the AD, the AD-CAA as well as of the control brains. Compared to controls AD brains had significantly more CMBs in the superior frontal, the inferior temporal, the rectus and the cinguli gyrus, and in the insular cortex. In AD-CAA brains CMBs were increased in all gyri with exception of the medial parietal gyrus and the hippocampus. AD-CAA brains showed a highly significant increase of CMBs in the inferior parietal gyrus ( $p$ value: 0.001 ) and a significant increase in the precuneus and the cuneus ( $p$ value: 0.01 ) compared to the AD brains. The differences in topographic distribution of CMBs between $\mathrm{AD}$ and AD-CAA brains should be further investigated on MRI in clinically suspected patients.
\end{abstract}

Key words: 7.0-tesla magnetic resonance imaging, topography of post-mortem cortical microbleeds, Alzheimer's disease, cerebral amyloid angiopathy (CAA)

Cerebral microbleeds are frequently detected on $\mathrm{T} 2 *_{-}$ weighted gradient-echo (GRE) magnetic resonance imaging (MRI) in patients with small-vessel diseases such as cerebral amyloid angiopathy (CAA) and lipohyalinosis [1]. They are also found in asymptomatic persons and in patients with various degrees of cognitive impairment $[2,3]$.

Cortical microbleeds (CMBs) are frequently associated with the clinical manifestation and the biochemical hallmarks of Alzheimer disease (AD) [4]. They are mainly observed in AD brains with severe CAA [5-6]. CAA is not only responsible for an increased number of CMBs, but also of lobar hematomas, cortical microinfarcts and severe white matter changes [7]. A set of validated criteria (termed the Boston criteria) has been established to diagnose CAA during life. The presence of multiple CMBs detected by GRE MRI has been shown to be highly specific for severe CAA in elderly patients in whom no other cause was found [8]. However, a definite diagnosis can only be made by neuropathological examination [9].

Less is known about the topographic distribution of CMBs in $\mathrm{AD}$ and in AD-CAA brains. CMBs in CAA brains appear to have a posterior predominance with spatial clustering [10]. Our previous study showed that CMBs were more widespread in the frontal sections at the

*Correspondence should be addressed to: Dr. J. L. De Reuck, Leopold II laan 96, BE-9000, Ghent, Belgium. Email: 
level of the head of the caudate nucleus, in the central sections at the level of the mamillary body and in the occipital sections behind the splenium corporis callosi of AD-CAA brains. In AD brains without CAA they only predominated in the central sections [11]. In another study CMBs were found to have a predilection for the parietal lobe in CAA brains [12].

The present post-mortem MRI study compares the prevalence of CMBs in twenty-two different cortical gyri of pure $\mathrm{AD}$ and of $\mathrm{AD}-\mathrm{CAA}$ brains mutually and to controls. The goal is to determine whether eventual differences could be helpful to investigate in a clinical setup.

\section{MATERIALS AND METHODS}

\section{Patients and Materials}

Thirty-two patients, followed-up at the Lille University Hospital underwent an autopsy. The final diagnosis was made according to the validated neuropathological criteria [13]. The cohorts consisted of 12 patients with $A D, 8$ with AD-CAA and 12 control brains, from individuals who had no clinical history of dementia or stroke (C). Also none of the AD patients with or without CAA had a stroke history. A previously obtained informed consent of the patients or from the nearest family allowed an autopsy for diagnostic and scientific purposes. The brain tissue samples were acquired from the Lille Neuro-Bank of the Lille University that is part of the Centres des Resources Biologiques and act as an institutional review board. The treatment of research participants is in accord with the ethical and other requirements in France, in which the research is conducted and specified by the INSERM U1171.

One fresh cerebral hemisphere was frozen at -80 degree Celsius for biochemical examination. The remaining hemisphere, the brainstem and most of the cerebellum were fixed in formalin for 3 weeks.

The brain samples with AD and AD-CAA were compared concerning the incidence of CMBs to the control group. Also the patients with pure AD were separately compared to those with AD-CAA.

\section{Neuropathological examination}

The diagnosis of $\mathrm{AD}$ was made according to a standard procedure examining samples from the primary motor cortex, the associated frontal, temporal and parietal cortex, the primary and secondary visual cortex, the cingulate gyrus, the basal nucleus of Meynert, the amygdaloid body, the hippocampus, basal ganglia, mesencephalon, pons, medulla and cerebellum. All slides from paraffin-embedded sections were stained by haematoxylin-eosin. A selected number of them were immune-stained for protein tau, $\beta$-amyloid, $\alpha$-synuclein, prion protein, TDP-43 and ubiquitin.

The brains were classified similar to the CERAD (Consortium to Establish a Registry for Alzheimer's Disease) criteria as AD-CAA, when a majority of $\beta$ amyloid stained vessels were present in at least three of the four examined samples and as not-CAA, when absent or scarce, in case of a few stained vessels in one or two slides [6].

A quantitative evaluation of the number of CMBs was performed on a standard coronal section of a cerebral hemisphere, at the level of the mamillary body according to a previous described method. Also the other cerebrovascular lesions were evaluated and compared between $\mathrm{AD}, \mathrm{AD}-\mathrm{CAA}$ and the control groups according to a previously described method [14].

The mean scores in $\mathrm{AD}$ and $\mathrm{AD}-\mathrm{CAA}$ brains were compared to the controls.

\section{MRI Examination}

Six coronal sections of a cerebral hemisphere from each brain were submitted to MRI: one at the prefrontal level in front of the frontal horn, one of the frontal lobe at the level of the head of the caudate nucleus, a central one near the mamillary body, a post-central one, a parietal one at the level of the splenium corporis callosi and one at the level of the occipital lobe.

We used a 7.0-tesla MRI Bruker BioSpin SA with an issuer-receiver cylinder coil of $72 \mathrm{~mm}$ inner diameter (Ettlingen, Germany), according to a previous described method [15]. The brain sections were first cleaned from the formalin fixation and afterwards placed in an adapted plastic box filled with salt free water, the size of which did not allow significant tissue movements. Three MRI sequences were used: a positioning sequence, a T2 sequence and a $\mathrm{T} 2 *$ sequence. The positioning sequence allowed determination of the three-directional position of the brain section inside the magnet. The thickness of the $\mathrm{T} 2$ images was $1 \mathrm{~mm}$. The field of view was a $9-\mathrm{cm}$ square slide that was coded by a 256 matrix giving a voxel size of $0.352 \times 0.352 \times 1 \mathrm{~mm}$. T2 weighted images were obtained by using RARE sequence (Rapid Acquisition with Relaxation Enhancement) with repetition time (TR), echo time (TE) and RARE factor of 2,500 ms, 33ms and 8 , respectively. The acquisition time of this sequence was $80 \mathrm{~s}$. The thickness of the T2* images was $0.20 \mathrm{~mm}$. The field of view was also a $9 \mathrm{~cm}$-square. It was coded by a 512 matrix, giving a voxel size of $0.176 \times 0.176 \times 0.2 \mathrm{~mm}$. This sequence was a GRE sequence with a short TR of 60 $\mathrm{ms}$ and TE of $22 \mathrm{~ms}$, a flip angle of $30^{\circ}$ and number of excitations of 20. The acquisition time of the sequence was $10 \mathrm{~min}$. 
Table 1. Brain regions and gyri of interest on magnetic resonance imaging

\begin{tabular}{llll}
\hline Frontal Lobe & Temporal Lobe & Parietal lobe & Occipital Lobe \\
\hline Frontalis inferior & Temporalis inferior & Postcentralis & Lingualis \\
Frontalis medius & Temporalis medius & Insula & Precuneus \\
Frontalis superior & Temporalis superior & Parietalis inferior & Cuneus \\
Precentralis & Hippocampus & Parietalis medius & Occipitotemporalis \\
Rectus & Dentatus & Parietalis superior & \\
Orbitalis & Parahippocampalis & Cinguli & \\
\hline
\end{tabular}

Our previous validation study showed a good correlation between CMBs detected on MRI and their neuropathological correlates [15].

The total number and the location of CMBs was determined in 22 gyri of each brain (table 1 ) by consensus evaluation by three observers (JDR, FA, ND) blinded to the neuropathological diagnosis and based on comparison of brain sections of an anatomical atlas [16]. The interrater reliability resulted in an interclass correlation coefficient of 0.81 . The mean values of CMBs in $\mathrm{AD}$ and AD-CAA brains were mutually compared and also to the controls.

\section{Statistical analyses}

Univariate comparisons of unpaired groups were performed with the Fisher's exact test for categorical data. The non-parametric Mann-Whitney U-test was used to compare continuous variables. The significance level, two-tailed, was set at $\leq 0.01$ for significant and $\leq 0.001$ for highly significant. Values set at $\leq 0.05$ and more than $>0.01$ were considered as marginal significant and not included as relevant due to the relative small sample sizes [17].

Table 2. Demographic and neuropathological features in controls and in Alzheimer patients without (AD) and with cerebral amyloid angiopathy (AD-CAA)

\begin{tabular}{llll}
\hline Items & Control $(\mathbf{n}=\mathbf{1 2})$ & AD $(\mathbf{n}=\mathbf{1 2})$ & AD-CAA (n= 8) \\
\hline Age: years (interquartile range) & $64(60-78)$ & $68(63-76)$ & $70(63-88)$ \\
Gender $(\%$ males) & 67 & 67 & 50 \\
Vascular risk factors (\%) & & & \\
Arterial hypertension & 17 & 42 & 38 \\
Diabetes & 17 & 17 & 25 \\
Hypercholesterolemia & 17 & 17 & 25 \\
Smoking & 0 & 8 & 13 \\
Antithrombotic drug use & 25 & 33 & 50 \\
Neuropathological lesions: mean values (SD) & & & \\
White matter changes & $0.3(0.8)$ & $0.8(0.8)$ & $1.3(1.3)$ \\
Lacunar infarcts & $0.0(0.0)$ & $0.0(0.0)$ & $0.3(0.7)$ \\
Territorial infarcts & $0.0(0.0)$ & $0.1(0.3)$ & $0.0(0.0)$ \\
Lobar haematomas & $0.0(0.0)$ & $0.0(0.0)$ & $0.0(0.0)$ \\
Cortical microinfarcts & $0.1(0.3)$ & $0.8(1.1)$ & $1.4(1.1) *$ \\
Cortical microbleeds & $0.1(0.3)$ & $0.4(0.7)$ & $1.1(0.6)^{*}$ \\
* = p value $\leq 0.01$ between the brains with Alzheimer disease associated with cerebral amyloid angiopathy, and the controls.
\end{tabular}




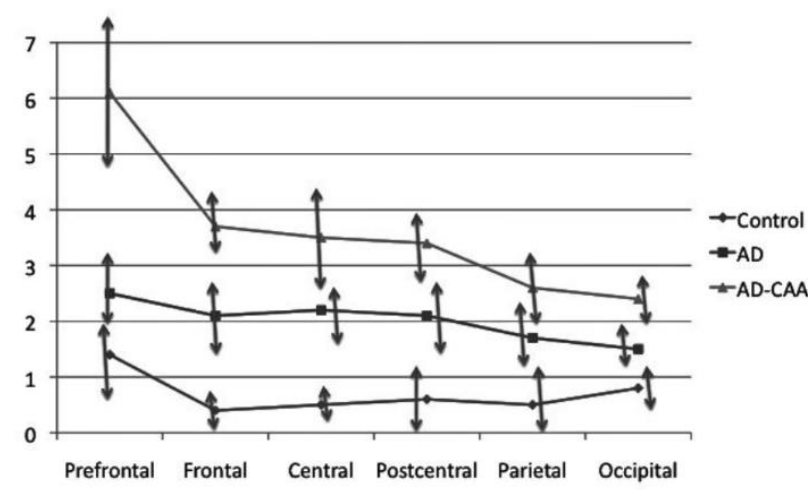

Figure 1. Mean numbers of cortical microbleeds in the six coronal sections on $\mathbf{T} 2$ *-weighted gradient-echo magnetic resonance imaging. An anterior-posterior decreasing gradient of the cortical microbleeds is observed in the Alzheimer brains with and without cerebral amyloid angiopathy as well as in the control brains.

\section{RESULTS}

The three comparison groups did not show any statistical differences according to age, gender distribution and vascular risk factors. However, on the neuropathological examination $\mathrm{CMBs}$ and cortical microinfarcts were significantly increased in the AD-CAA group compared to the control ( $\mathrm{p}$ value $\leq 0.01$ ), but not to the AD brains. No statistical differences were observed for the other types of cerebrovascular lesions (table 2).

As a whole there was on MRI a trend of more CMBs in the prefrontal section of the $\mathrm{AD}$, the AD-CAA as well as of the control brains (Fig. 1).

The mean values of $\mathrm{CMBs}$ in $\mathrm{AD}$ brains were significantly increased in the superior frontal, the inferior temporal, the rectus and the cingulate gyri, and in the insular cortex, compared to the control brains ( $\mathrm{p}$ value $\leq$ 0.01). When comparing the AD-CAA brains to the controls all gyri had significantly more CMBs ( $\mathrm{p}$ value $\leq$ 0.001 ) with exception of the medial parietal gyrus and the hippocampus. Comparison of the AD-CAA to the AD brains without CAA showed in the former a statistically highly increased number of CMBs in the inferior parietal gyrus ( $\mathrm{p}$ value $\leq 0.001$ ) (Fig. 2), and also a statistical increase in the precuneus and cuneus $(\mathrm{p}$ value $\leq 0.01)$ (Fig. 3) (Table 3).
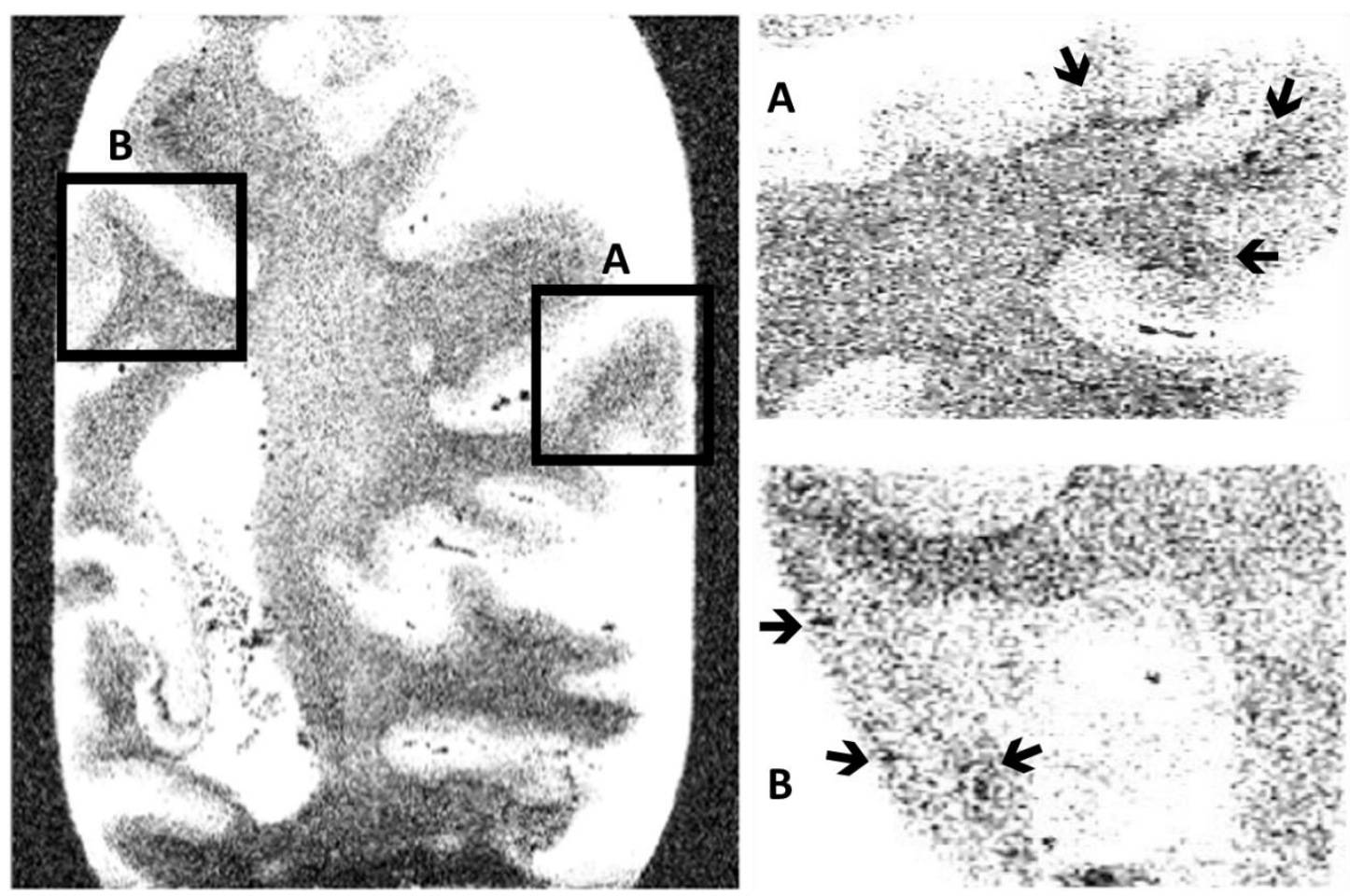

Figure 2. Parietal coronal section. Cortical microbleeds on $\mathrm{T} 2 *$-weighted gradient-echo magnetic resonance imaging of a whole coronal parietal brain section and more in detail in the gyrus parietalis inferior (A) and in the precuneus (B). The arrows indicate the presence of some cortical microbleeds. 

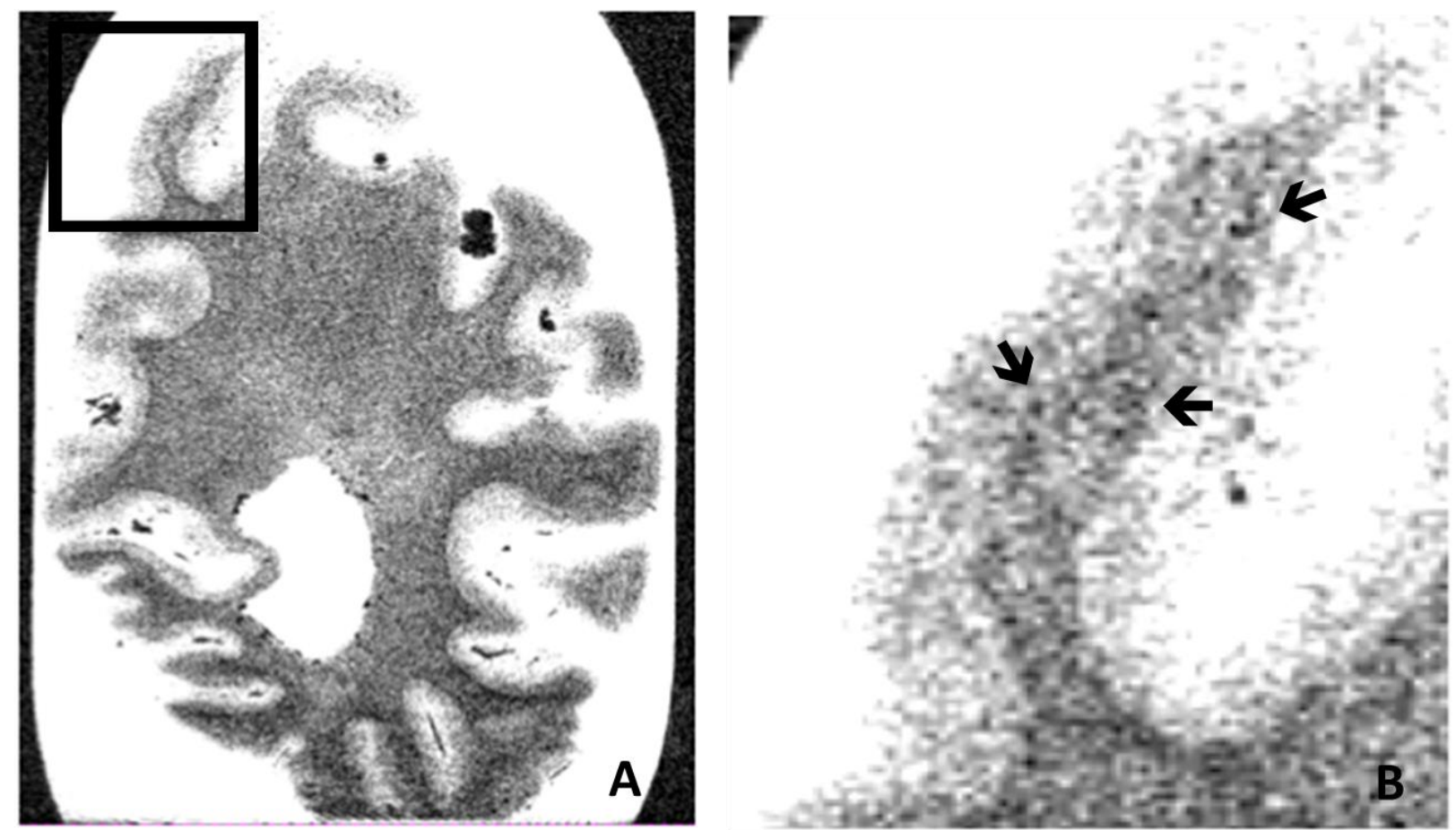

Figure 3. Occipital coronal section. Cortical microbleeds on $\mathrm{T} 2 *$-weighted gradient-echo magnetic resonance imaging of a whole coronal occipital brain section (A) and more in detail in the cuneus (B). The arrows indicate the presence of some cortical microbleeds.

\section{DISCUSSION}

The present study shows significant topographic differences in the number of CMBs between control, $\mathrm{AD}$ and $\mathrm{AD}-\mathrm{CAA}$ brains. CMBs have a more widespread distribution and are more frequent in $\mathrm{AD}-\mathrm{CAA}$ than in $\mathrm{AD}$ brains compared to controls, as previously shown [11]. In addition there is a gyral predilection for CMBs in the inferior parietal gyrus, the precuneus and the cuneus of AD-CAA compared to AD brains. This is in concordance with their posterior predominance found in another study [10]. Also on functional MRI impaired blood flow responses in CAA are more evident using a task to activate the occipital than the frontal lobe, consisting with a gradient of increased vascular amyloid severity from frontal to occipital lobe [18].

The gyrus parietalis inferior is composed of the gyrus supramarginalis and the gyrus angularis. They are involved in the perception of emotions in facial stimuli and concerned with language, mathematic operations and body image [19]. The precuneus is involved in memory tasks and directing in space [20]. The cuneus is involved in basic visual processing [21]. It is, however, thoughtful whether the $\mathrm{CMBs}$ in these predilection areas of $\mathrm{AD}-\mathrm{CAA}$ brains have a significant impact on the clinical course of the disease, as overall CMBs predominate in the prefrontal areas of the AD-CAA, the AD as well as of the control brains.

Although there are quantitative differences there is a remarkable anterior-posterior decreasing gradient of CMBs in the AD, the AD-CAA as well as in the control brains. This must be due to anatomical factors such as the larger development of the frontal lobe compared to the parietal and occipital lobe [22].

The main purpose of the present study was to determine whether differences in the topography of CMBs could helpful for diagnosing additional CAA in $\mathrm{AD}$ patients. The preferential increase of CMBs in the gyrus parietalis inferior, the precuneus and the cuneus should be further investigated on MRI in AD patients suspected from CAA. It should be determined whether these findings could have an additional value in the clinical evaluation of these patients.

Disclosure of conflicts of interest: The authors declare no financial or other conflicts of interest. 
Table 3. Mean numbers of cortical microbleeds with standard deviations between bracquets in the different gyri of control and Alzheimer brains without (AD) and with cerebral amyloid angiopathy (AD-CAA)

\begin{tabular}{lllll}
\hline Gyrus & $\mathbf{C}(\mathbf{n}=\mathbf{1 2})$ & $\mathbf{A D}(\mathbf{n}=\mathbf{1 2})$ & AD-CAA $(\mathbf{n}=\mathbf{8})$ & $\begin{array}{l}\text { P value } \\
\text { AD/AD-CAA }\end{array}$ \\
\hline Frontalis inferior & $0.8(0.9)$ & $2.3(2.7)$ & $4.8(2.9)^{* *}$ & 0.03 \\
Frontalis medius & $1.2(1.4)$ & $1.9(1.4)$ & $4.3(2.5)^{*}$ & 0.03 \\
Frontalis superior & $1.2(1.4)$ & $3.1(1.8)^{*}$ & $6.7(3.4)^{* *}$ & 0.02 \\
Precentralis & $1.1(1.5)$ & $2.1(1.3)$ & $2.8(0.8)^{*}$ & 0.27 \\
Rectus & $0.4(0.7)$ & $1.5(1.2)^{*}$ & $4.4(3.8)^{* *}$ & 0.07 \\
Orbitalis & $0.8(1.1)$ & $2.1(2.0)$ & $4.3(1.6)^{* *}$ & 0.02 \\
Temporalis inferior & $0.4(1.0)$ & $2.6(3.0)^{*}$ & $3.4(2.6)^{* *}$ & 0.30 \\
Temporalis medius & $0.5(0.8)$ & $1.9(1.9)$ & $3.7(2.9)^{* *}$ & 0.16 \\
Temporalis superior & $0.6(1.1)$ & $1.5(1.2)$ & $3.9(3.2)^{* *}$ & 0.06 \\
Hippocampus & $0.4(0.8)$ & $0.8(0.8)$ & $1.1(1.6)$ & 1.0 \\
Dentatus & $0.7(1.2)$ & $1.9(2.1)$ & $2.4(1.7)^{*}$ & 0.38 \\
Parahippocampalis & $0.5(1.1)$ & $1.0(1.6)$ & $2.0(1.2)^{*}$ & 0.05 \\
Postcentralis & $0.7(0.9)$ & $2.3(2.6)$ & $2.9(1.6)^{* *}$ & 0.18 \\
Insula & $0.0(0.0)$ & $1.8(2.7)^{*}$ & $2.4(1.8)^{* *}$ & 0.18 \\
Parietalis inferior & $0.3(0.4)$ & $0.7(0.6)$ & $2.9(2.2)^{* *}$ & $0.001^{* *}$ \\
Parietalis medius & $1.2(1.4)$ & $1.7(1.0)$ & $2.9(2.6)$ & 0.34 \\
Parietalis superior & $0.6(0.5)$ & $1.7(1.6)$ & $2.7(2.3)^{*}$ & 0.31 \\
Cinguli & $0.4(0.6)$ & $1.5(1.7)^{*}$ & $3.0(2.0)^{* *}$ & 0.02 \\
Lingualis & $0.3(0.9)$ & $1.4(2.7)$ & $2.9(2.2)^{* *}$ & 0.03 \\
Precuneus & $0.6(1.0)$ & $0.9(1.6)$ & $3.3(2.8)^{*}$ & $0.01^{*}$ \\
Cuneus & $0.5(0.9)$ & $0.8(1.5)$ & $3.2(2.6)^{*}$ & $0.01^{*}$ \\
Occipitotemporalis & $0.6(1.0)$ & $2.4(2.2)$ & $4.6(3.0)^{* *}$ & 0.18 \\
& & & & \\
\hline
\end{tabular}

$*=\mathrm{p}$ value $\leq 0.01 ; * *=\mathrm{p}$ value $\leq 0.001$ comparing $\mathrm{AD}, \mathrm{AD}-\mathrm{CAA}$ and control brains.

\section{References}

[1] Greenberg SM, Vernooij MW, Cordonnier C, Viswanathan A, Al-Shari Salman R, Warach S, et al (2009). Cerebral microbleeds: a guide to detection and interpretation. Lancet Neurol, 8: $165-174$.

[2] Cordonnier C, Al-Shahi Salman R, Wardlaw J (2007). Spontaneous brain microbleeds, systematic review, subgroup analyses and standards for study design and reporting. Brain, 130: 1988-2003.

[3] Cordonnier C, van der Flier WM, Sluimer JD, Leys D, Barkhof F, Scheltens Ph (2006). Prevalence and severity of microbleeds in a memory clinic setting. Neurology, 66: 1356-1360.

[4] Goos JD, Kester MI, Barkhof F, Klein M, Scheltens $P$, van der Flier WM (2009). Patients with Alzheimer disease with multiple microbleeds: relation with cerebrospinal fluid biomarkers and cognition. Stroke, 40: 3455-
3460.

[5] Nakata-Kudo Y, Mizuno T, Yamada K, Shiga K, Yoshikawa K, Mori S, et al (2006). Microbleeds in Alzheimer disease are more related to cerebral amyloid angiopathy than to cerebrovascular disease. Dement Geriatr Cogn Disord, 22: 8-14.

[6] Ellis RJ, Olichney JM, Thal LJ, Mirra SS, Morris JC, Beekly BS, et al (1996). Cerebral amyloid angiopathy in the brains of patients with Alzheimer's disease: the CERAD experience, Part XV. Neurology, 46: 1592-1596.

[7] De Reuck J, Deramecourt V, Cordonnier C, Leys, Maurage CA, Pasquier F (2011). The impact of cerebral amyloid angiopathy on the occurrence of cerebrovascular lesions in demented patients with Alzheimer features: a neuropathological study. Eur J Neurol, 18: 913-918.

[8] Vismanathan A, Greenberg SM (2011). Cerebral amyloid angiopathy in the elderly. Ann Neurol, 70: 871-880. 
[9] Knudsen KA, Rosand J, Karluk D, Greenberg SM (2001). Clinical diagnosis of cerebral amyloid angiopathy. Neurology, 56: 537-539.

[10] Rosand J, Muzikansky A, Kumar A, Wisco JJ, Smith EE, Betensky RA, et al (2005). Spatial clustering of hemorrhages in probable cerebral amyloid angiopathy. Ann Neurol, 58: 459- 462.

[11] De Reuck J, Cordonnier C, Deramecourt V, Auger F, Durieux N, Bordet R, et al (2013). Microbleeds in postmortem brains of Alzheimer disease: a T2*weighted gradient-echo 7.0 T magnetic resonance imaging study. Alzheimer Dis Assoc Disord, 27: 162-165.

[12] Lee SH, Kim SM, Kim N, Yoon BW, Roh JK (2007). Cortico-subcortical distribution of microbleeds is different between hypertension and cerebral amyloid angiopathy. J Neurol Sci. 258: 111-114.

[13] De Reuck J (2012). The significance of small cerebral bleeds in neurodegenerative dementia syndromes. Aging Dis, 4: 307-312.

[14] De Reuck J, Deramecourt V, Cordonnier C, Leys D, Pasquier F, Maurage C-A (2011). Prevalence of small cerebral bleeds in patients with a neurodegenerative dementia: a neuropathological study. J Neurol Sci, 30: 63-66.

[15] De Reuck J, Auger F, Cordonnier C, Deramecourt V, Durieux N, Pasquier F, et al (2011). Comparison of $7.0-\mathrm{T} 2 *$-magnetic resonance imaging of cerebral bleeds in post-mortem brain sections of Alzheimer patients with their neuropathological correlates. Cerebrovasc Dis 2011, 31: 511-517.
[16] Roberts M, Hanaway J. Atlas of the human brain in section. Philadelphia. Lea \& Febiger, 1970.

[17] Sproull NL. "Hypothesis testing". Handbook of Research Methods: A Guide for Practitioners and Students in the Social Science ( $2^{\text {nd }}$ ed.). Lanham. Scarecrow Press, 2002: 49-64.

[18] Peca S, McCreary CR, Donaldson E, Kumarpillai G, Shobha N, Sanchez K, et al (2013). Neurovascular decoupling is associated with severity of cerebral amyloid angiopathy. Neurology, 81: 1659-1665.

[19] Radua J, Philips ML, Russell T, Lawrence N, Marshall N, Kalidindi S, et al (2010). Neural response to specific components of fearful faces in healthy and schizophrenic adults. Neuroimage, 49: 939-946.

[20] Cavanna AE, Trimble MR (2006). The precuneus: a review of its functional anatomy and behaviour correlates. Brain, 129: 564-583.

[21] Rodrigues G, Morbelli S, Brugnolo A, Calvini P, Girtler N, Piccardo A, et al (2005). Global cognitive impairment should be taken into account in SPECT-neuropsychology correlations: the example of verbal memory in very mild Alzheimer's disease. Eur J Nucl Med Mol Imaging, 32: 1186-1192.

[22] Semendeferi K, Damasio H, Frank R (1997). The evolution of the frontal lobes: a volumetric analysis based on three-dimensional reconstructions of magnetic resonance scans of human and ape brains. J Hum Evol, 32: 375-388. 